\title{
Evaluation of Surface Smoothness Detected by a Periodontal Probe
}

\author{
Gul Atilla and Sukru Kandemir
}

(Received 10 December 1993 and accepted 19 July 1994)

Key words: smoothness, roughness, roughness value (Ra), sense of touch

\begin{abstract}
An investigation was designed to determine whether or not the required smoothness has been reached. This is still a controversial subject among clinicians.

Ten cylindrical metal samples of equal sizes with various degrees of smoothness were prepared, and their degrees of smoothness were assessed in a blind manner by 14 periodontists, who were asked to group them as smooth or rough using a periodontal probe. Along with the metal samples, the roughness of root surfaces smoothed in clinics was also evaluated using a Profilometer.

The average value defined as a smooth surface on a max.-min. scale was $1.86 \pm 0.42$, and values above 3.57 were considered to be rough. Consequently, decisions taken using the sense of touch about the smoothness of a surface were confirmed to be reliable for clinical use.
\end{abstract}

\section{Introduction}

Cementum is a calcified tissue which enables the periodontal ligament fibers to attach to the roots of teeth. The presence of microbial plaque and calculus on periodontally diseased cementum has been observed. Furthermore, mineralization of the cementum surface as a result of periodontal disease is increased, resulting in loss of collagen cross-banding on the cementum surface ${ }^{[1-5]}$. It has been concluded that these pathological changes are due to loss of biological compatibility on the cementum surface ${ }^{[1-3]}$.

The ultimate objective of scaling ( $\mathrm{Sc}$ ) and root planing ( $\mathrm{Rp}$ ) is to render the treated root surface biologically compatible for reattachment with host periodontal tissues ${ }^{[6-9]}$. In a clinical situation, root surfaces to be treated are identified by subgingival probing of roughness, and therapy is terminated upon detection of the disappearance of roughness ${ }^{[10-13]}$. Root surface smoothness is considered to be a sign indicating the removal of microbial plaque, calculus, hypermineralized cementum and endotoxin from the root surface ${ }^{[14]}$. This criterion is currently accepted as the basis for successful periodontal treatment. The other reason for root planing is the prevention of subgingival bacteria retention ${ }^{[14]}$. Contrary to this opinion, Khatiblou et al. ${ }^{[15]}$ have claimed that the degree of root surface smoothness dose not play a significant role in pocket reduction and attachment gain.

On the other hand, root surface smoothness can be assessed by the sense of touch using a periodontal probe or the sharp edge of a curette. Unfortunately, a practical instrument that can evaluate the smoothness of a root surface quantitatively is not yet available. The degree of surface smoothness that can be assessed by the sense of touch and whether or not the required smoothness has been reached are still a matter of controversy among clinicians.

Our present study was designed primarily to determine how much roughness is acceptable to define a surface as being smooth by periodontists, and to obtain the roughness values of cementum considered to be smooth using the sense of touch. 


\section{Materials and Methods}

Ten cylindrical metal samples of equal diameter were prepared at different roughness values utilizing standard roughening operations. Different disk abrasive waterproofs (Struers, Denmark) with silicon carbide grains from 80 to 1000 were used with a Knuth Rotor (KNUTH ROTOR, Struers, Denmark) for this operation. The roughness values ( $\mathrm{Ra}$ ) of these metal samples were estimated in a blind manner by 14 periodontists, who were requested to separate them into two groups, rough or smooth, utilizing a Starlite MG No.1 Periodontal Probe. Taking into account the border values between the smooth and rough groups, 10 new samples with very similar roughness values were then prepared. The periodontists were then requested once again to separate these samples into two groups, rough or smooth.

For clinical application, 10 extracted teeth were used. All of the teeth had single roots and at least moderate periodontitis prior to extraction. Sc and $\mathrm{Rp}$ were carried out using a sharp Gracey 1-2 curette until smoothness was reached in a $3 \times 5-\mathrm{mm}$ area on the cementum surface of each extracted tooth (Fig. 1).

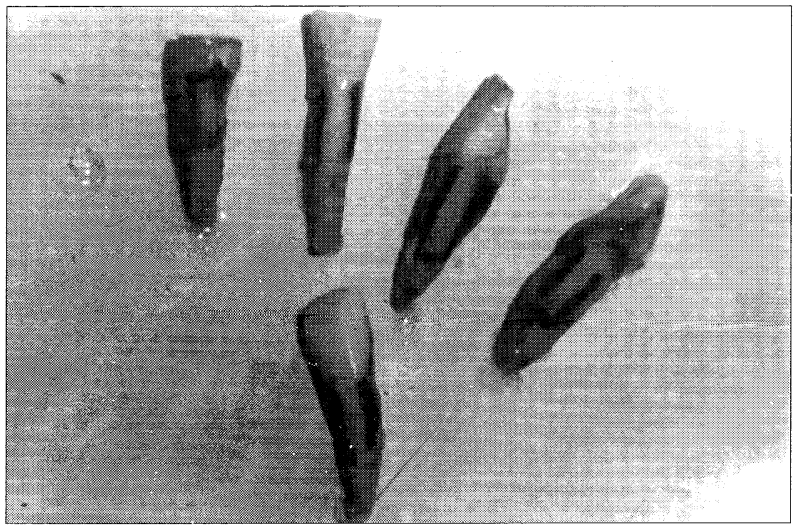

Fig. 1 The cementum surfaces of the extracted teeth

\section{Roughness Measurements:}

Upon completion of the Sc and Rp procedures, and after all the cementum surfaces had been rinsed in running tap-water, the surface roughness was measured with a Profilometer (Perthen Mahr, Germany) ${ }^{[16]}$, which was calibrated against a standard. The profilometer used for making surface roughness measurements is shown in Fig. 2. To measure the roughness, a standard has been developed by the American Standards Association (ASA B46.1 - 1962)(ISO R468). In the present study, a max.-min. scale was used to measure the surface roughness ${ }^{[16]}$. On this max.-min. scale (Fig. 3), the distance between the maximum and minimum points at the examined surface gives the roughness value.

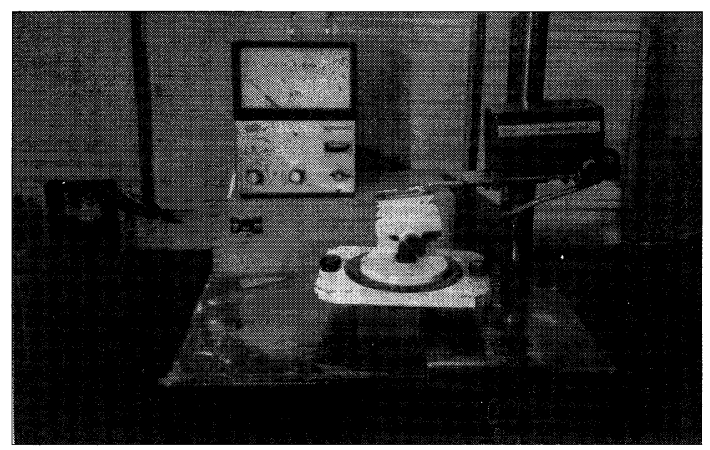

Fig. 2 Profilometer instrument

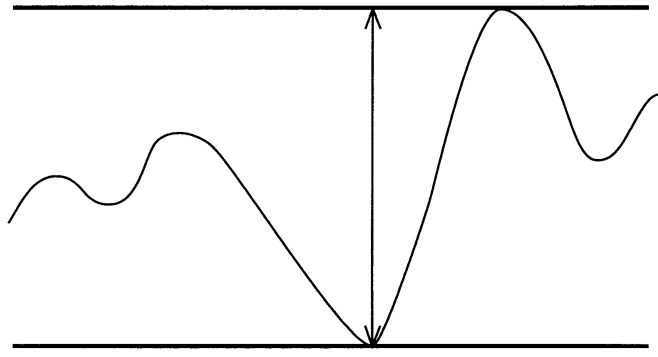

Fig. 3 Diagrammatic illustration of max. - min. scale 
The stylus of the Profilometer was run in 5 parallel scans a $0.5-\mathrm{mm}$ intervals for a length of $1.5 \mathrm{~mm}$ along each surface ${ }^{[16]}$. The values obtained on the scale were expressed in micrometers. The sensitivity of the Profilometer is $0.01 \mu \mathrm{m}$.

\section{Results}

When the metal samples had been grouped according to the max.-min. scale, all of the samples whose roughness values $(\mathrm{Ra})$ were below 2.12 were considered to be smooth by the periodontists, and the samples whose Ra were above 3.57 were considered to be rough (Fig. 4). The metal samples with Ra below 2.12 were accepted as smooth by $100 \%$ of the periodontists. On the other hand, $79 \%, 72 \%, 43 \%, 7 \%$ of the periodontists accepted as smooth $\mathrm{Ra}=2.12, \mathrm{Ra}=2.61, \mathrm{Ra}=3.20$ and $\mathrm{Ra}=3.57$, respectively (Fig. 4 ).

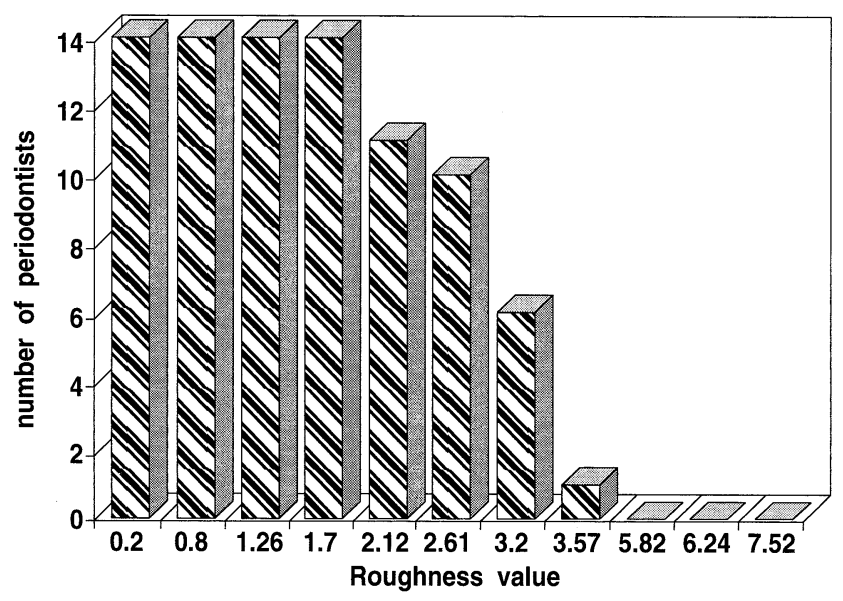

Fig.4 Distribution of the samples accepted as smooth among the periodontists

In a Rp carried out by a certain periodontist, it was observed that smoothness in a $3 \times 5-\mathrm{mm}$ area on the root surface was achieved after about 20 strokes.

The average of the roughness value on the root surface smoothed by $\mathrm{Sc}+\mathrm{Rp}$ was found to be $1.86 \pm$ $0.42(\min .=1.66, \max .=2.36)$.

\section{Discussion}

In the present study, an in vitro model was employed to facilitate standardization of the experimental conditions. The Rp was performed under ideal conditions with direct access to the test surfaces.

The smoothness and roughness evaluations of metal samples by the 14 periodontists who took part in our study varied between 2.12 and 3.57 according to the max.-min. scale. All of the surfaces with values below 2.12 were accepted as smooth and those possessing values above 3.57 were considered to be rough . The $\mathrm{Ra}$ of the root surfaces smoothed by $\mathrm{Sc}+\mathrm{Rp}$ was $1.86 \pm 0.42$.

In our reference study, the results obtained here could not be compared because we have not encountered any study which has determined the roughness value of a root surface smoothed by $\mathrm{Sc}+\mathrm{Rp}$.

According to the result obtained from both the teeth and metal surfaces, the average roughness value which may be defined as smooth was $1.86 \pm 0.42$, and values above 3.57 as rough. The fact that the sizes of bacteria may vary between $0.2-10 \mu \mathrm{m}$ should be taken into consideration, since this roughness may act to retain the bacteria ${ }^{[17]}$. As stated by Listgarten ${ }^{[18]}$, the thickness of subgingival microbial plaque in the gingival sulcus may change between 1 to 60 bacteria, whereas in adult periodontitis the thickness of plaque may reach up to $400 \mu \mathrm{m}$. According to our findings, the roughness on the root surface causes bacterial adhesion, but this roughness is not convenient enough to allow the bacteria to increase. Thus, it is clearly 
understood that the formation of supragingival plaque or marked retention of bacteria on the root surface is required to form pathogenic subgingival plaque.

It is generally accepted that treatment is successful after efficient plaque control and Sc $+\mathrm{Rp}$ in all clinical studies ${ }^{[19-21]}$. According to the results of our study, the roughness value of the metal surfaces whose smoothness was determined by the sense of touch was 2.12 on average. This roughness value was similar to that of the root surfaces, and is not capable of allowing subgingival plaque to form after supragingival plaque has been well removed. Contrary to Khatiblou et al.'s opinion ${ }^{[15]}$, our findings support the opinions of other researchers ${ }^{[10-13]}$ who also suggest that $\mathrm{Rp}$ can achieve smoothness on the cementum surface. Consequently, decisions taken according to the sense of touch about the smoothness of a surface have been confirmed to be reliable for clinical use.

\section{Conclusion}

In this study, surface roughness evaluations were made in detail by periodontists. The findings confirmed that decisions taken according to the sense of touch about surface smoothness are reliable for utilization in clinics.

\section{References}

[ 1 ] Aleo, J.J., DeRenzis, F.A. and Farber, P.A.: In vitro attachment of human gingival fibroblasts to root surfaces, J. Periodontol., 46, 639-645, 1975

[2] Hatfield, C.G. and Baumhammers, A.: Cytotoxic effects of periodontally involved surfaces of human teeth, Arch. Oral Biol., 16, 465-468, 1971

[ 3 ] Olson, R.H., Adams, D.F. and Layman, D.L.: Inhibitory effect of periodontally diseased root extracts on the growth of human gingival fibroblasts, J. Periodontol., 56, 592-596, 1985

[4] Selvig, K.A.: Ultrastructural changes in cementum and adjacent connective tissue in periodontal disease, Acta Odontol. Scand., 24, 459-500, 1966

[ 5 ] Selvig, K.A. and Hals, E.: Periodontally diseased cementum studied by correlated microradiography, electron probe analysis and electron microscopy, J. Periodontol. Res., 12, 419-429, 1977

[6] Badersten, A., Nilveus, R. and Egelberg, J.: Effect of nonsurgical periodontal therapy. II. Severly advanced periodontitis, J. Clin. Periodontol., 11, 63-76, 1984

[ 7 ] Cercek, J., Kiger, R., Garrett, S. and Egelbert, J.: Relative effects of plaque control and instrumentation on the clinical parameters of human periodontal disease. J. Periodontol., 10, 46-53, 1983

[ 8 ] Lang, N.: Indications and rationale for nonsurgical periodontal therapy. Inter. Dent. J., 33, 127-135, 1983

[9] Morrison, E., Ramfjord, S. and Hill, R.: Short-term effects of initial, nonsurgical periodontal treatment(hygienic phase), J. Clin. Periodontol., 7, 199-206, 1980

[10] Garret, J.S.: Root planing: A perspective, J. Periodontol., 48, 553-557, 1977

[11] Jones, W.A. and O'Leary, T.J.: The effectiveness of in vivo root planing in removing bacterial endotoxin from the roots of periodontally involved teeth, J. Periodontol., 49, 337-342, 1978

[12] O'Leary, T.J. and Kafrawy, A.H.: Total cementum removal: A realistic objective ?, J. Periodontol., 54, 221-226, 1983

[13] Baylas, H.: Evaluation of endotoxin in the involved cementum tissue,(dissertation) 1988, Izmir

[14] Wilson, S.B.: Scaling and root planing. In: Periodontics, Vol. II, 650-652, Grant, D.A., Stern, I.B., Listgarten, M.A.,eds., Mosby Company, U. S. A., 1988

[15] Khatiblou, F.A. and Ghodssi,A.: Root surface smoothness or roughness in periodontal treatment (A clinical study), J. Periodontol., 54, 365-367, 1983

[16] Leitao, J. and Hegdahl, T.: On the measuring of roughness, Acta. Odontol. Scan., 39, 379-384, 1981

[17] Bilgehan, H.: General Microbiology and Immunology, 8-10, Bilgehan Press, Izmir, 1983

[18] Listgarten, M.A.,eds., Structure of the microbial flora associated with periodontal health and disease in man. A light and electron microscopic study, J. Periodontol., 47, 1-18, 1976 
[19] Axelsson, P. and Lindhe, J.: Effect of controlled oral hygiene procedures on caries and periodontal disease in adults, J. Clin. Periodontol., 5, 133-151, 1978

[20] Badersten, A., Nilveus, R. and Egelberg, J.: Effect of nonsurgical periodontal therapy. II. Moderately advanced periodontitis, J. Clin. Periodontol., 8, 57-72, 1981

[21] Suomi, J.D., Greene, J.C. and Vermillion, J.R.: The effect of controlled oral hygiene procedures on the progression of periodontal disease in adults: Results after third and final year, J. Periodontol., 42, 152160, 1971 